\title{
IMMUNOLOGY
}

\section{A knock-in mouse lights up killer T cells}

Chitirala, P. et al. elife 9, e58065 (2020)

Cytotoxic T lymphocytes (CTLs) are a type of white blood cell that the adaptive immune system sets to work against invaders. That includes foreign foes, such as bacteria and viruses, as well as familiar ones-CTLs can also identify and kill cancerous cells, raising the possibility that these cells could prove useful in immunotherapies.

As the name suggests, these killer $\mathrm{T}$ cells are cytotoxic - they kill problematic cells via the coordinated release of toxic molecules contained within cytotoxic granules: perforin, a protein that perforates the cell membrane of the target, and granzymes, proteases that use the resulting pores to enter the cell and initiate its programmed cell death, or apoptosis.

At least's that's how things work in vitro, where CTLs from both humans and mice have been well studied. In that context, the killer T cells are fast and efficient at serially eliminating their targets. Cell cultures, however, exist in isolation: in vivo, CTLs perform their duties alongside the rest of the immune system.

"These T cells never act alone," says Jens Rettig, a researcher at Saarland University in Homburg, Germany. They do their job at initiating the killing of targeted cells, but at the same time CTLs release a number of signaling molecules to call other immune cells to the scene, such as the macrophages that clean up the mess left behind. They also function more slowly in vivo than they do in vitro, suggesting that there are rate limits that need be determined.

The tools and techniques available to study CTLs in vivo, however, have been limited. Some of the current methods, for example, involve removing CTLs from a mouse, exogenously labelling the whole cells with a fluorescent marker, and then re-injecting the manipulated cells back into the animal. In vivo approaches can also be complicated by overexpression of CTL genes of interest, limiting the biological relevance to the animal.

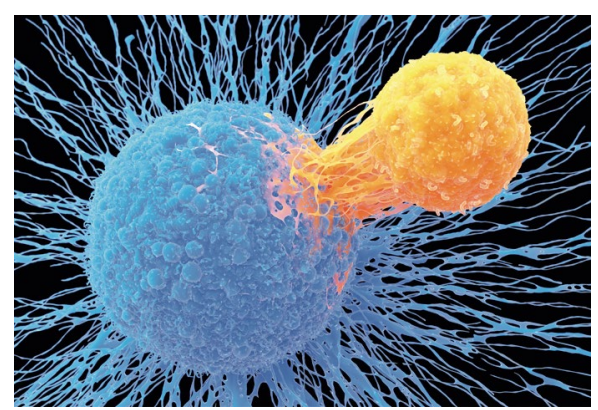

Cytotoxic T lymphocytes, depicted in orange here, are cell-killing immune agents. A new knock-in mouse gives them a fluorescent teal tinge to study how they operate in vivo. Credit: Roger Harris / Science Photo Library / Getty

Rettig and his lab recently took a different, endogenous approach, using CRISPR-Cas9 to knock-in a monomeric teal fluorescent protein (mTFP) localized to the locus of granzyme B (GzMB). This is one of the protease components of the cytotoxic granules, which Rettig refers to as the 'bullets' of CTLs. "In principle, we will be able to not only follow the killing process in real time, but we should also be able to follow the release of a single bullet," says Rettig.

The teal fluorescent protein they used is bright under a 2-photon microscope and suitable for longitudinal studies, but it's a bit bulky—about $27 \mathrm{kDA}$, to GzmB's 30 . To keep the fluorescent tag from interfering with the protease's expression and/or function, the team linked it to the $G z m B$ gene in such a way that the two molecules would be cleanly cleaved apart by the acidic environment of the cytotoxic granules. "So we have the fluorophore, and we have the free granzyme B," says Rettig.

After confirming that GzmB was indeed free to function unimpeded while the fluorescent tag kept track of when and where it was expressed, and that expression levels of GzmB matched those of wild-type mice, the team put their granzyme B-mTFP knock-in mice to work. With in vitro preparations from the transgenic mice, they were able to follow the priming process and fusion kinetics as CTLs made contact with targets and fired their cytotoxic granule bullets. . Abnormalities in that fusion process are linked to a number of human diseases, says co-first author Hsin-Fang Chang, the dynamics of which had been difficult to observe before. Moving in vivo, they were able to follow single CTLs over the course of three weeks as they identified and eliminated allografted tissue implanted in the anterior chamber of the eye. "This is exactly what we want to follow," says Rettig, "And we want to follow that over an extended period of time with non-damaging microscopy."

Though the fluorescent cells could in theory be imaged anywhere else a microscope can penetrate in the body, the eye, as an optical system itself, has the imaging benefit of being transparent. "This is taking advantage of that natural, perfect optical system and just doing our imaging," says Rettig. With a combination of stimulated emission depletion (STED) and 2-photon microscopy, they are planning to increase the penetration depth, with the goal to resolve not only cells but the smaller organelles and compartments within them.

Going forward, they hope the fluorescent granzyme B-mTFP knock-mice will have translational relevance as well. "This mouse really enables in vivo studies," says Rettig. "You can cross it with any disease model, and then look at the effect and see exactly what step is going wrong."

Ellen P. Neff

Published online: 17 August 2020 https://doi.org/10.1038/s41684-020-0634-X 